\title{
Stress evolution in Si during low-energy ion bombardment
}

\section{Citation}

Ishii, Yohei, Charbel S. Madi, Michael J. Aziz, and Eric Chason. 2014. "Stress Evolution in Si During Low-Energy lon Bombardment." J. Mater. Res. 29 (24) (November 25): 2942-2948. doi:10.1557/jmr.2014.350.

\section{Published Version}

doi:10.1557/jmr.2014.350

\section{Permanent link}

http://nrs.harvard.edu/urn-3:HUL.InstRepos:22968433

\section{Terms of Use}

This article was downloaded from Harvard University's DASH repository, and is made available under the terms and conditions applicable to Open Access Policy Articles, as set forth at http:// nrs.harvard.edu/urn-3:HUL.InstRepos:dash.current.terms-of-use\#OAP

\section{Share Your Story}

The Harvard community has made this article openly available.

Please share how this access benefits you. Submit a story.

Accessibility 


\title{
Stress evolution in Si during low energy ion bombardment
}

\author{
Yohei Ishii $^{1,2}$, Charbel S. Madi ${ }^{3,4}$, Michael J. Aziz ${ }^{3}$ and Eric Chason ${ }^{1}$ \\ ${ }^{1}$ Brown University, School of Engineering, Providence, Rhode Island, 02912, USA \\ ${ }^{2}$ Hitachi High Technologies America, Inc, Semiconductor Equipment Division, Dallas TX 75261-2208 \\ ${ }^{3}$ Harvard School of Engineering and Applied Sciences, Cambridge Massachusetts, 02138, USA \\ ${ }^{4}$ Department of Physics, American University of Beirut, Beirut 1107 2020, Lebanon
}

\begin{abstract}
Measurements of stress evolution during low energy argon ion bombardment of Si have been made using a real-time wafer curvature technique. During irradiation, the stress reaches a steady state compressive value that depends on the flux and energy. Once irradiation is terminated, the measured stress relaxes slightly in a short period of time to a final value. To understand the ion-induced stress evolution and relaxation mechanisms, we account for the measured behavior with a model for viscous relaxation that includes the ion-induced generation and annihilation of flow defects in an amorphous $\mathrm{Si}$ surface layer. The analysis indicates that bimolecular annihilation (i.e., defect recombination) is the dominant mechanism controlling the defect concentration both during irradiation and after the cessation of irradiation. From the analysis we determine a value for the fluidity per flow defect.
\end{abstract}

Keywords: ion-solid interactions, defects, radiation effects

Corresponding Author: Eric Chason (Eric_Chason@Brown.edu) 


\section{INTRODUCTION}

Low energy ion beams are used widely in thin film processing [1] and stress induced by the beam may affect the film properties [2]. To understand the processes controlling stress generation and relaxation, we have used a wafer curvature technique to measure stress evolution under different irradiation conditions. We find that the ion-induced stress depends on the flux, suggesting that defectdependent relaxation processes are playing a role in controlling it. This is in contrast with previous studies of stress evolution in amorphous Si during high energy $(\mathrm{MeV})$ ion irradiation [3-5] in which the stress depended primarily on the total number of ions (fluence) but not the rate of impingement (flux).

There has been previous experimental work on stress in the low-energy regime performed on crystalline metals [6-10]. Dahmen et al. [6] studied stress in $\mathrm{Cu}$ due to noble gas ion bombardment and explained the steady state stress as a balance between stress induced by implantation and the sputter removal of the bombarded layer. Chan et al. [7] also found a compressive steady-state stress in $\mathrm{Cu}$, but found that the stress relaxes to a tensile state after stopping the ion bombardment. They explained this [10] with a model that includes volumetric relaxation around mobile defects in addition to ion implantation and sputtering. Work on Pt [8] showed that heavier or higher-energy ions tend to create tensile stress, while lighter ions induce compressive stress. The results were explained by a competition between local melting and the creation of ion-induced interstitial defects.

Kalyanasundaram et al. [11] calculated the stress generated by low-energy bombardment of Si by molecular dynamics (MD) simulation and showed that the ion implantation process and subsequent damage production could produce large compressive stresses that were proportional to the number of implanted ions. However, because of the short time scales studied by MD, any effects of relaxation after implantation could not be observed.

Covalent materials can behave very differently than elemental metals because they typically become amorphous during bombardment, allowing relaxation mechanisms (e.g., viscous flow) that don't 
occur in crystalline systems. Volkert suggested a model for stress evolution during irradiation based on a fluidity (reciprocal of the viscosity, $\eta$ ) that is directly proportional to the ion flux $[3,4]$, i.e., $1 / \eta=f / \eta_{\text {rad }}$, where $f$ is the flux and $\eta_{\text {rad }}$ is a flux-independent parameter that may depend on the material, temperature, and ion beam [12]. Proportionality between fluidity and flux is consistent with the observation that the stress relaxation depends on the fluence but not on the flux; in other words the amount of viscous flow per ion does not depend on the rate at which the ions impinge on the surface. This approach has been successful in describing irradiated materials in the high ion energy regime where electronic stopping is the dominant mechanism of ion energy loss $[3,5,12]$. The similar values of $\eta_{\text {rad }}$ found for many materials and conditions further suggests it doesn't depend strongly on the particular material. Mayr et al. [13] used MD to calculate the radiation-induced fluidity over a range of energies and found good agreement with measurements on $\mathrm{Si}$ and $\mathrm{SiO}_{2}$ as well as some amorphous metals. Viscous relaxation in amorphous Si in the absence of irradiation was successfully modeled by Witvrouw and Spaepen [14] as being governed by the concentrations and mobilities of flow defects. During thermal annealing, these defects could annihilate by various mechanisms so that the viscosity could evolve as well as the stress.

\section{EXPERIMENT AND RESULTS}

In the work described here, stress evolution was monitored in situ during ion bombardment using a Multibeam Optical Stress Sensor (MOSS) wafer curvature-measurement technique [15]. Details of the configuration of the sputtering system and stress measurement technique have been described previously [7]. Measurements were made on polished $\operatorname{Si}(100)$ wafers $(100 \mu \mathrm{m}$ thickness x $1.25 \mathrm{~cm}$ (width) x 1.25 $\mathrm{cm}$ (length)) with $\mathrm{Ar}^{+}$ions at normal incidence angle and room temperature from a $1 \mathrm{~cm}$ Kaufmann source (base pressure $1.0 \times 10^{-7}$ Torr) using energies of 600 and $900 \mathrm{eV}$. To investigate whether there was contamination from the surroundings onto the sample surface during irradiation, test samples were 
measured by energy dispersive X-ray spectrometry and found to have no measurable contamination from the chamber.

The measured curvature $(\kappa)$ is interpreted using the Stoney equation $[16,17]$

$$
\sigma d=\frac{M_{s} h_{s}^{2} \kappa}{6}
$$

where $M_{s}$ and $h_{s}$ are the biaxial modulus and thickness of the unmodified substrate, respectively. $\sigma$ refers to the in-plane stress in the implanted layer averaged over the thickness of the implanted region, $d$. This thickness is small compared to $h_{s}$ and corresponds to the range over which stress is produced by the ion beam. Values of $d$ for the different ion energies are given in table 1, estimated from SRIM calculations [18] for the ion range plus twice the longitudinal straggle. This has been confirmed by cross-sectional transmission electron microscopy as a good estimate for the thickness of the amorphous layer for $250 \mathrm{eV}$ argon ion bombardment of Si at normal incidence [19].

A representative measurement of the time-dependence of the stress is shown in figure 1 . The magnitude of the compressive stress initially increases very rapidly (in the first $15 \mathrm{~s}$ ) but then reaches a plateau for fluences between approximately $6 \times 10^{15}-3 \times 10^{16}$ ions $/ \mathrm{cm}^{2}$ (indicated by arrow and expanded in inset). We attribute this to the amorphization of the layer; data obtained at other fluxes also showed plateaus or peaks that occur at approximately the same fluence. As the fluence increases further, the stress becomes increasingly compressive and eventually reaches a steady state that does not change with continued bombardment.

When the beam is turned off (at approximately $1000 \mathrm{~s}$ ), the stress relaxes to a slightly less compressive value, by about $50 \mathrm{MPa}$ out of 800 . The stress change occurs over a very short period after the beam is turned off, reaching a final value in less than $10 \mathrm{~s}$. Because the relaxation was fast relative to our acquisition rate, the measurement of the relaxation rate (discussed below) has large error bars. To confirm that the stress relaxation is not due to thermal transients when the beam is turned off, a sample that had been previously ion bombarded was heated to $45 \mathrm{deg} \mathrm{C}$, which is larger than any temperature 
change measured during ion bombardment using thermocouples. The stress changed by less than $10 \mathrm{MPa}$ due to heating, which indicates that the stress relaxation cannot be attributed to thermal expansion effects but instead corresponds to changes in the intrinsic stress of the layer. Stress due to ion-induced temperature gradients was also ruled out by calculation of their contribution [20].

Curvature measurements were made during bombardment and relaxation at ion energies of 600 and $900 \mathrm{eV}$ over the flux range $1.25-6.25 \times 10^{14} \mathrm{ions} / \mathrm{cm}^{2} \mathrm{~s}$. For all the fluxes and energies measured, a sequence of stress evolution behavior similar to that shown in figure 1 was observed, i.e., saturation at a steady-state and rapid relaxation when the beam was terminated. The value of the steady-state compressive stress as a function of ion flux is shown in figure 2 for different values of the ion energy. Note that for both ion energies, the steady-state stress is observed to become more compressive at higher flux.

\section{ANALYSIS AND STRESS MODEL}

To model the data, we assume that the stress we measure comes from the amorphous surface layer (and not, for instance, from an implanted crystalline layer below it). To confirm this, we measured the change in curvature after selectively removing the amorphous layer using a Wright etch [21]. This reduced the stress-induced curvature by $70 \%$, indicating that most of the measured stress is in the amorphous Si. In addition, molecular dynamics (MD) simulations of stress from low energy ion bombardment of $\mathrm{Si}$ [11] show that the stress is confined primarily to the amorphous layer. We also assume that the stress is uniform throughout the layer so that we can model the average stress for comparison with the results obtained from the curvature measurements.

We consider the evolution of the average stress $(\sigma)$ as a balance between competing generation and relaxation mechanisms, using an approach similar to the one used previously for higher energy bombardment $[3,12,13]$; this has also been shown to be reasonable for ion energies as low as $3 \mathrm{keV}[22]$. 
We use the following ordinary differential equation to describe processes of stress generation and
relaxation:
\[ \frac{d \sigma}{d t}=f \Sigma_{0}-\frac{f Y \Omega}{d} \sigma-\frac{M_{f}}{6} \frac{1}{\eta} \sigma \]

The first term on the right hand side corresponds to the generation of stress by the incoming ions, where $f$ is the ion flux and $\Sigma_{\mathrm{o}}$ is the stress generated per unit fluence (flux $\times$ time). The second term is due to the effect of sputter removal of the stressed layer (where $\Omega$ is the atomic volume and $Y$ is the sputtering yield, i.e., atoms sputtered away per incident ion). Physically, this corresponds to a moving boundary condition as the top surface is eroded and new regions of film are implanted at the bottom of the layer. For high energy ions this term is generally not important because the sputter yield is relatively low and the range is large. For low energies, however, this term cannot be neglected. In this case, sputter removal can lead to the stress saturating at a steady-state value even in the absence of any stress relaxation mechanisms [6]. The third term corresponds to relaxation of the stress in the layer by Newtonian viscous flow, as previously discussed by others $[3-5,12,13,23]$. The rate of relaxation is dependent on the inverse of the viscosity (i.e., fluidity) and the stress. The dependence of the fluidity on ion irradiation is discussed further below. $M_{f}$ is the biaxial modulus of the stressed layer.

In the steady-state $(\mathrm{d} \sigma / \mathrm{dt}=0)$, the stress is predicted to have the value

$$
\sigma_{s s}=\frac{f \Sigma_{o}}{\frac{f Y \Omega}{d}+\frac{M_{f}}{6 \eta}}
$$


In the absence of relaxation in the layer (i.e., $1 / \eta=0$ ), the steady-state stress would be independent of flux with a value of $\sigma_{s s}=\Sigma_{o} d / Y \Omega$. In the presence of fluidity, however, the steady-state stress is predicted to depend on the rate of relaxation.

Previous work at higher energies $[3,5,13]$ was explained with a radiation-induced fluidity that was proportional to the ion flux, i.e., $1 / \eta=f / \eta_{\text {rad }}$. Using this form for the fluidity, however, leads to a predicted steady-state stress from eq. 3 that is independent of the flux. This is not consistent with our measurements, and indicates that there must be other relaxation mechanisms involved. These other relaxation mechanisms must be radiation dependent because the stress in the film does not continue to relax significantly after the beam is turned off.

To explain this behavior, we invoke a model of viscous relaxation in amorphous Si proposed by Witvrouw and Spaepen [14]. The fluidity during thermal relaxation is taken to be proportional to the concentration of "flow defects" -- such as broken bonds -- that enable rearrangement of the covalent network:

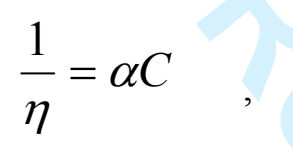

where $\mathrm{C}$ is the concentration of flow defects in units $\mathrm{of}^{-3}$ and $\alpha$ is a constant specifying the fluidity per unit concentration. Witvrouw and Spaepen used this to explain the relaxation of stress induced by thermal expansion mismatch when a layer of amorphous Si on a crystalline Si substrate was heated (i.e., with no ion irradiation). They were able to interpret the time-evolution of the viscosity in terms of the changing concentration of defects in the material, which were annihilated either by unimolecular processes (at sinks) or bimolecular processes (by recombination of defects with each other).

In the current work, we add the consideration of two ion beam-induced effects that were not present in the thermal relaxation studies: flow defects are created by the ion beam, and layers containing 
defects can be removed by sputter erosion. Combining these mechanisms gives the following ordinary differential equation for the defect concentration in the implanted layer:

$$
\frac{d C}{d t}=\frac{f C_{0}}{d}-\frac{f Y \Omega}{d} C-D_{1} C-D_{2} C^{2}
$$

The first term on the right hand side corresponds to the creation of $\mathrm{C}_{\mathrm{o}}$ flow defects per ion. The second term corresponds to removing defects by sputtering away the layers containing them. The third and fourth terms correspond, respectively, to unimolecular and bimolecular annihilation with the respective coefficients $D_{1}$ and $D_{2}$.

These ion-induced defect kinetics can explain why the stress reaches a steady-state when the beam is on but does not relax completely when the beam is turned off. During ion bombardment, the defects reach a steady-state concentration due to a balance between beam-induced defect creation and defect annihilation. When the beam is turned off, the concentration of flow defects decreases and therefore the fluidity decreases, turning off the stress relaxation process. This also tells us that the defect recombination must be relatively fast compared to the stress relaxation or else the stress would continue to decay even after the beam is turned off.

We can obtain the model's prediction for the steady-state stress vs. flux from eq. 3 using the value for the fluidity when the defect concentration has reached its steady-state value $\left(1 / \eta=\alpha C_{s s}\right)$. The dependence of $C_{s s}$ on flux depends on which removal mechanism in eq. 5 is dominant; we determine this by setting $d C / d t$ equal to zero and considering the effect of each term . If defect removal is dominated by the sputter removal rate, then $C_{s s}$ is independent of the flux and equal to $C_{o} / Y \Omega$. If bimolecular annihilation dominates, then $C_{s s}$ depends on the square root of flux $\left(\left(f C_{o} / D_{2} d\right)^{1 / 2}\right)$. Finally, if unimolecular annihilation dominates, then $C_{s s}$ is proportional to the flux $\left(f C_{d} D_{l} d\right)$. Note that if $C_{s s}$ were proportional to the flux then the steady-state stress (from eq. 3) would be independent of the flux (because all the terms 
would depend linearly on the flux). This is in contradiction to the experimental results which means that unimolecular annihilation cannot be the dominant defect removal mechanism during irradiation.

There are therefore two possible defect annihilation mechanisms (either sputter removal or bimolecular annihilation) that we can compare with our experimental results to determine which dominates annihilation during irradiation and controls the steady-state defect concentration (and hence the steady-state stress). We can obtain the predictions of these two cases for the steady-state stress by evaluating eq. 3 with the appropriate steady-state concentration $\left(C_{\delta} / Y \Omega\right.$ for sputter removal and $\left(f C_{d} / D_{2} d\right)^{1 / 2}$ for bimolecular annihilation). When we compare these with the measured flux-dependence of the steady-state stress, however, both mechanisms give reasonable agreement. This means that the steady-state stress measurement alone cannot be used to determine which mechanism is dominant.

In order to differentiate between these possible mechanisms, we can also consider the measurements of stress relaxation when the ion beam is turned off. To do this, we solve eqs. 2 and 5 for the simultaneous evolution of the stress and defect concentration when the flux is set equal to zero. The initial conditions for this solution are that the defect concentration and stress have their steady-state values.

We first consider the possibility that sputter removal determines the steady-state defect concentration during irradiation (i.e., $\mathrm{C}_{\mathrm{ss}}=\mathrm{C}_{\mathrm{o}} / \mathrm{Y} \Omega$ ). We then fit the relaxation data to determine the kinetic parameters when the ion beam is turned off $(f=0)$. Assuming that unimolecular annihilation is dominant when irradiation is turned off, we obtain parameters $\alpha=9.11 \times 10^{-39} \mathrm{~cm}^{3} / \mathrm{Pa}-\mathrm{s}$ and $D_{l}=$ $0.00513 / \mathrm{s}$. However, if we use these parameters to evaluate the defect removal term in eq. 5 during irradiation, we find that $D_{l} C_{s s}$ is larger than the sputter removal term $\left(f Y \Omega C_{s s} / d\right)$. This contradicts our initial assumption that sputter removal is dominant during irradiation and indicates that it cannot be the mechanism that determines $C_{s s}$. If we instead assume that bimolecular annihilation is dominant when irradiation is turned off, we obtain parameters $\alpha=3.32 \times 10^{-35} \mathrm{~cm}^{3} / \mathrm{Pa}-\mathrm{s}$ and $D_{2}=1.52 \times 10^{-24} \mathrm{~cm}^{3} / \mathrm{s}$. Again, using these parameters to evaluate the defect removal term in eq. 5 during irradiation indicates that 
$D_{2} C_{s s}{ }^{2}$ is larger than the sputter removal term. Since both these possibilities result in defect removal terms that are larger than that from sputtering, sputter removal cannot be the dominant mechanism that determines $C_{s s}$ during irradiation at the fluxes studied here. Physically, this also agrees with our expectation that the flow defects are short-lived and their concentration decays rapidly after the beam is terminated.

Alternatively, we consider bimolecular annihilation to be the mechanism that dominates the defect annihilation during irradiation so that $C_{s s}=\left(f C_{d} / D_{2} d\right)^{1 / 2}$. We simultaneously fit the data for relaxation when irradiation is turned off to three different scenarios in which the the flow defects are annihilated by unimolecular $\left(D_{l}\right)$ mechanism, a bimolecular $\left(D_{2}\right)$ mechanism or both $\left(D_{l}\right.$ and $\left.D_{2}\right)$. The parameters obtained from the fitting indicate that in all cases $D_{2} C^{2}>>D_{1} C$ so that the bimolecular annihilation has the largest rate when the beam is turned off. In this case there is no inconsistency between our assumptions regarding the dominant annihilation mechanism during irradiation and the deductions from the relaxation kinetics when the beam is turned off. By considering all the possible combinations of mechanisms, we therefore conclude that bimolecular annihilation is the dominant mechanism controlling the defect concentration both when the beam is on and when it is turned off, for the range of fluxes studied here. This conclusion is qualitatively consistent with the conclusions of studies of topographic pattern formation during sputter erosion, which find that morphology evolution is dominated by structural rearrangements within the irradiated material rather than by sputter erosion $[24,25]$.

Using this conclusion that bimolecular annihilation controls the defect concentration, we analyze the data during both irradiation and relaxation to obtain values for the model parameters. In this case, the flux-dependent steady-state stress is given by:

$$
\sigma_{s s}=\frac{1}{\frac{M_{f}}{6} \sqrt{\frac{C_{0}}{d}}}\left(\frac{\Sigma_{0} \sqrt{D_{2}}}{\alpha}\right) \sqrt{f}
$$


Fitting this equation to the experiments requires only one composite fitting parameter, $\beta$, which is equal to $\Sigma_{o}\left(D_{2}\right)^{1 / 2} / \alpha$. The results of the fitting for this parameter are given in table 1 for each energy. Other parameters used for the calculations $\left(d, Y\right.$ and $\left.C_{o}\right)$ are determined using SRIM. The atomic volume is taken as $\Omega=2.0 \times 10^{-23} \mathrm{~cm}^{3} /$ atom and $\mathrm{M}_{\mathrm{f}}=135 \mathrm{GPa}$ [4]. The results of the model calculation using these parameters are shown as the solid lines in figure 2 for each ion energy.

We determine the individual parameters $D_{2}, \Sigma_{o}$ and $\alpha$ from the relaxation measurements. When the flux is set to zero (at time $t=0$ ) then $d C / d t=-D_{2} C^{2}$ under the assumption that bimolecular annihilation is the dominant relaxation mechanism. The defects decay with the form

$$
C(t)=\frac{1}{\frac{1}{C_{s s}}+D_{2} t}
$$

from their initial concentration of $C_{s s}$. Using this concentration evolution with eqs. 2 and 4 , the corresponding stress is predicted to decay with the form:

$$
\sigma(t)=\sigma_{s s}\left[\frac{\frac{1}{C_{s s}}}{\frac{1}{c_{s s}}+D_{2} t}\right]^{\frac{\alpha M_{f}}{6 D_{2}}}
$$

where the stress has its steady-state value $\left(\sigma_{s s}\right)$ at $t=0$.

Calculations of the simultaneous evolution of the stress and the defect concentration during relaxation are shown in figure 3 using parameters obtained from fitting the data in figure 1 (after the beam is turned off) to eq. 8 . When the beam is terminated, the calculated defect concentration (fig $3 \mathrm{a}$ ) rapidly decays due to bimolecular annihilation. Consequently, the calculated stress (solid line in fig 3b) only decreases for a short time because the fluidity drops rapidly as the number of flow defects decreases. The 
measured stress relaxation data from figure 1 are shown on the same figure for comparison. The values obtained from the non-linear least squares fitting are $\alpha=5.74 \times 10^{-30} \mathrm{~cm}^{3} / \mathrm{Pa}-\mathrm{s}, D_{2}=1.86 \times 10^{-17} \mathrm{~cm}^{3} / \mathrm{s}$ and $\Sigma_{o}=-1.07 \times 10^{-14} \mathrm{GPa}-\mathrm{cm}^{2}$.

The results from fitting the full set of measurements of steady-state stress and stress relaxation under different conditions are summarized in table 1. The values and error estimates of $\Sigma_{o}, D_{2}$ and $\alpha$ were obtained from the results of the fitting at different fluxes for each energy. As noted earlier, the relaxation data have a large degree of variability so that the average values have relatively large error bars.

In the analysis described above, we have only compared the model with the steady-state stress and relaxation data and not the buildup of the compressive stress during ion bombardment. Although the model equations predict the stress evolution during this period, the layer is amorphizing which means that there are presumably other effects involved that are not included in the model. However, we note that if we consider the stress data only after the plateau (corresponding to amorphized Si), then the model does provide reasonable agreement with the measurements during irradiation.

\section{DISCUSSION}

To assess the plausibility of the parameters obtained from fitting the model to the data, we compare their values with those from other studies. A model for defect recombination [26] relates the parameter $\mathrm{D}_{2}$ to the defect diffusivity and recombination radius. From the wide range of measured values for defect (interstitial and vacancy) diffusion in $\mathrm{Si}\left(10^{-11}-10^{-30} \mathrm{~cm}^{2} / \mathrm{s}\right)[27-29]$, we would expect the value of $D_{2}$ to be in the range of $10^{-17}-10^{-37} \mathrm{~cm}^{3} / \mathrm{s}$. This is consistent with what we find from our modeling.

The value of $\Sigma_{o}$ indicates that the amount of stress generated by implantation of the equivalent of a monolayer of ions $\left(6.8 \times 10^{14} \mathrm{ions} / \mathrm{cm}^{2}\right)$ would be approximately $-5.4 \mathrm{GPa}$ if there were no viscous relaxation. For comparison, Kalyanasundaram et al. [11] used MD to calculate the stress induced by $\mathrm{Ar}^{+}$ ions with energy of $700 \mathrm{eV}$ over a cubic region with an edge length of $5.43 \mathrm{~nm}$. For the same monolayer 
ion fluence, their calculations predict that the average stress saturates at approximately $-1.6 \mathrm{GPa}$. The depth that they averaged over is approximately the same as the thickness of the implanted region that we used. Note that MD simulations only model short time periods so that they also do not include viscous relaxation effects. Finally, the value of $\alpha$ predicts the amount of fluidity for each flow defect to have an average value of $2.39 \times 10^{-30} \mathrm{~cm}^{3} / \mathrm{Pa}$-s . The value of this parameter could not be obtained from previous studies of stress relaxation.

The reasonable agreement with the data suggests that the model can adequately explain stress evolution in Si during ion bombardment in terms of relaxation by ion-induced flow defects. However, as noted earlier, the relaxation kinetics change when the beam is turned off. In addition, if the ion beam is turned on again after the stress has relaxed, the stress may initially become less compressive before going back to its steady-state value [20] - similar effects have been seen in the high energy regime [4]. Both these observations suggest that there may be other relaxation mechanisms that have not been included in our model (e.g., structural relaxation) that require further study.

\section{CONCLUSION}

In summary, we have investigated in situ stress evolution in Si (001) during and after low energy ion bombardment. The dependence of the steady-state stress on flux requires relaxation mechanisms beyond the radiation-induced fluidity used to explain measurements at higher energies. We have therefore developed a model that includes relaxation by ion-induced flow defects and that produces a value for the amount of fluidity per flow defect. In the future, more extensive MD simulations of viscous relaxation that include defect recombination may be able to demonstrate these relaxation processes and others not included in our model.

We thank Karl Ludwig and Joy Perkinson for helpful discussions. Research at Brown was supported by the U.S. Department of Energy, Office of Basic Energy Sciences, Division of Materials 
Sciences and Engineering under Award DE-FG02-01ER45913. Portions of the manuscript preparation for EC were supported by DE-SC0008799. Research at Harvard was supported by NSF-DMR-1409700.

\section{REFERENCES}

1. E. Chason, S.T. Picraux, J.M. Poate, J.O. Borland, M.I. Current, T. Diaz de la Rubia, D.J. Eaglesham, O.W. Holland, M.E. Law, C.W. Magee, J.W. Mayer, J. Melngailis and A.F. Tasch: Ion beams in silicon processing and characterization, J. Appl. Phys. Rev. 81, 6513 (1997).

2. N. V. Medhekar, W. L. Chan, V. B. Shenoy and E. Chason: Stress-enhanced pattern formation on surfaces during low-energy ion-bombardment, J. Phys.: Condens. Matter, 21, 224021 (2009).

3. C. A. Volkert: Stress and plastic flow in silicon during amorphization by ion bombardment, J. Appl. Phys, 70, 3521 (1991).

4. C. A. Volkert and A. Polman: Radiation-enhanced plastic flow of covalent materials during ion irradiation, Mat. Res. Soc. Symp. Proc, 235, 3 (1992).

5. E. Snoeks, A. Polman and C. A. Volkert: Densification, anisotropic deformation, and plastic flow of $\mathrm{SiO}_{2}$ during $\mathrm{MeV}$ heavy ion irradiation, Appl. Phys. Lett, 65, 2487 (1994).

6. K. Dahmen, M. Giesen, J. Ikonomov, K. Starbova and H. Ibach: Steady-state surface stress induced in noble gas sputtering, Thin Solid Films, 428, 6 (2003).

7. W. L. Chan, E. Chason and C. Iamsumang: Surface stress induced in $\mathrm{Cu}$ foils during and after low energy ion bombardment, Nucl. Instrum. Methods Phys. Res. B, 257, 428 (2007).

8. W. L. Chan, K. Zhao, N. Vo, Y. Ashkenazy, D. G. Cahill and R. S. Averback: Stress evolution in platinum thin films during low-energy ion irradiation, Phys. Rev. B, 77, 205405 (2008).

9. A. Debelle, G. Abadias, A. Michel and C. Jaouen: Stress field in sputtered thin films: Ion irradiation as a tool to induce relaxation and investigate the origin of growth stress, Appl. Phys. Lett, 84, 5034 (2004).

10. W. L. Chan and E. Chason: Stress evolution and defect diffusion in $\mathrm{Cu}$ during low energy ion irradiation: Experiments and modeling, J. Vac. Sci. Technol. A, 26, 44 (2007). 
11. N. Kalyanasumdaram, M. C. Moore, J. B. Freund and H. T. Johnson: Stress evolution due to medium-energy ion bombardment of silicon, Act. Mat, 54, 483 (2006).

12. M. L. Brongersma, E. Snoeks, T. van Dillen, and A. Polman: Origin of MeV ion irradiationinduced stress changes in $\mathrm{SiO}_{2}$, J. Appl. Phys. 88, 59 (2000).

13. S. G. Mayr, Y. Ashkenazy, K. Albe and R. S. Averback: Mechanisms of radiation-induced viscous flow: role of point defects, Phys. Rev. Lett. 90, 055505 (2003)

14. A. Witvrouw and F. Spaepen: Viscosity and elastic constants of amorphous Si and Ge, J. Appl. Phys, 74, 7154 (1993).

15. E. Chason: A kinetic analysis of residual stress evolution in polycrystalline thin films, Thin Solid Films 526, 1 (2012).

16. G. G. Stoney: The tension of metallic films deposited by electrolysis, Proc. R. Soc. (London) A, 82, 172 (1909)

17. L. B. Freund and S. Suresh, Thin Film Materials (Cambridge University Press, Cambridge, UK, 2003).

18. J. F. Ziegler and J. P. Biersack, SRIM-2008, IBM Co., Yorktown, NY, 1984-2008.

19. C. S. Madi, H. B. George and M. J. Aziz: Linear stability and instability patterns in ion-sputtered silicon, J. Phys. Condens. Matt, 21, 224010 (2009).

20. C. S. Madi: Linear Stability and Instability Patterns in Ion Bombarded Silicon Surfaces, Ph.D thesis, Harvard University, 2012.

21. M. W. Jenkins, J. Electrochem. Soc. Solid-State Sci and Tech: New preferential etch for defects in silicon-crystals, 124, 757 (1977).

22. H.B. George, Y. Tang, X. Chen, J. Li, J.W. Hutchinson, J.A. Golovchenko and M.J. Aziz: Nanopore fabrication in amorphous Si: Viscous flow model and comparison to experiment, J. Appl. Phys. 108, 014310 (2010).

23. M. L. Brongersma, E. Snoeks and A. Polman: Temperature dependence of MeV heavy ion irradiation-induced viscous flow in $\mathrm{SiO}_{2}$, Appl. Phys. Lett, 71, 1628 (1997). 
24. A. Norris, J. Samela, L. Bukonte, M. Backman, F. Djurabekova, K. Nordlund, C.S. Madi, M.P. Brenner and M.J. Aziz, Nature Communications 2, 276 (2011).

25. C.S. Madi, E. Anzenberg, K.F. Ludwig, and M.J. Aziz, Phys. Rev. Lett. 106, 066101 (2011); Erratum 110, 069903

26. K. Tai, R.S. Averback, P. Bellon, Y. Ashkenazy, and B. Stumphy: Temperature dependence of irradiation-induced creep in dilute nanostructured Cu-W alloys, J. Nucl. Mat, 422, 8 (2012).

27. S. Coffa and S. Libertino: Room-temperature diffusivity of self-interstitials and vacancies in ionimplanted Si probed by in situ measurements, Appl. Phys. Lett, 73, 3369, (1998).

28. P. M. Fahey, P. B. Griffin and J. D. Plummer: Point-defects and dopant diffusion in silicon, Rev. Mod. Phys, 61, 289 (1989).

29. G. B. Bronner and J. D. Plummer: Gettering of gold in silicon - a tool for understanding the properties of silicon interstitials, J. Appl. Phys, 61, 5286 (1987). 


\section{TABLE AND FIGURE CAPTIONS}

Table 1. Model parameters obtained from fitting the steady-state stress and stress relaxation data and from SRIM calculations.

Fig 1. Stress evolution during ion irradiation (in the region labeled beam on, $3.0 \times 10^{14} \mathrm{ions} / \mathrm{cm}^{2} \mathrm{~s}, 900 \mathrm{eV}$ ) and after beam is turned off. Negative stress values are compressive. The inset shows an expanded view of the stress plateau that is attributed to amorphization of the surface.

Fig. 2. Steady-state stress vs flux for ion bombardment with energies of $600 \mathrm{eV}$ and $900 \mathrm{eV}$. Lines are results of model calculations described in text.

Fig. 3. a) Model calculation of defect concentration in steady-state (beam on) and during relaxation (beam off). b) Model calculation of corresponding stress (smooth red line) from defect concentration. The model parameters were chosen to fit the data in figure 1 (shown as the black line for comparison). 
Tables and figures

Table 1. Model parameters obtained from fitting the steady-state stress and stress relaxation data and from SRIM calculations.

\begin{tabular}{|c|c|c|c|c|c|c|c|}
\hline \multirow{2}{*}{ Energy } & $\begin{array}{c}\beta \\
\left(\times 10^{-3}\right. \\
\left.\mathrm{GPa}^{2}(\mathrm{~s}-\mathrm{cm})^{1 / 2}\right)\end{array}$ & $\begin{array}{c}\Sigma_{\mathrm{o}} \\
\left(\times 10^{-15}\right. \\
\left.\mathrm{GPa}-\mathrm{cm}^{2}\right)\end{array}$ & $\begin{array}{c}D_{2} \\
\left(\times 10^{-18}\right. \\
\left.\mathrm{cm}^{3} / \mathrm{s}\right)\end{array}$ & $\begin{array}{c}\alpha \\
\left(\times 10^{-30}\right. \\
\left.\mathrm{cm}^{3} / \mathrm{Pa}-\mathrm{s}\right)\end{array}$ & $\begin{array}{c}d \\
(\mathrm{~nm})\end{array}$ & $Y$ & $\begin{array}{c}C_{0} \\
\text { vacancies/ion }\end{array}$ \\
\hline \multirow{2}{*}{$600 \mathrm{eV}$} & -9.52 & $\begin{array}{c}-8.64 \pm \\
10.7\end{array}$ & $\begin{array}{c}10.2 \pm \\
1.20\end{array}$ & $\begin{array}{c}2.40 \pm \\
2.37\end{array}$ & 5 & 0.412 & 18.4 \\
\hline $900 \mathrm{eV}$ & -8.08 & $\begin{array}{c}-7.27 \pm \\
2.51\end{array}$ & $\begin{array}{c}6.89 \pm \\
7.23\end{array}$ & $\begin{array}{c}2.38 \pm \\
2.12\end{array}$ & 6 & 0.547 & 27.0 \\
\hline
\end{tabular}


Figure 1

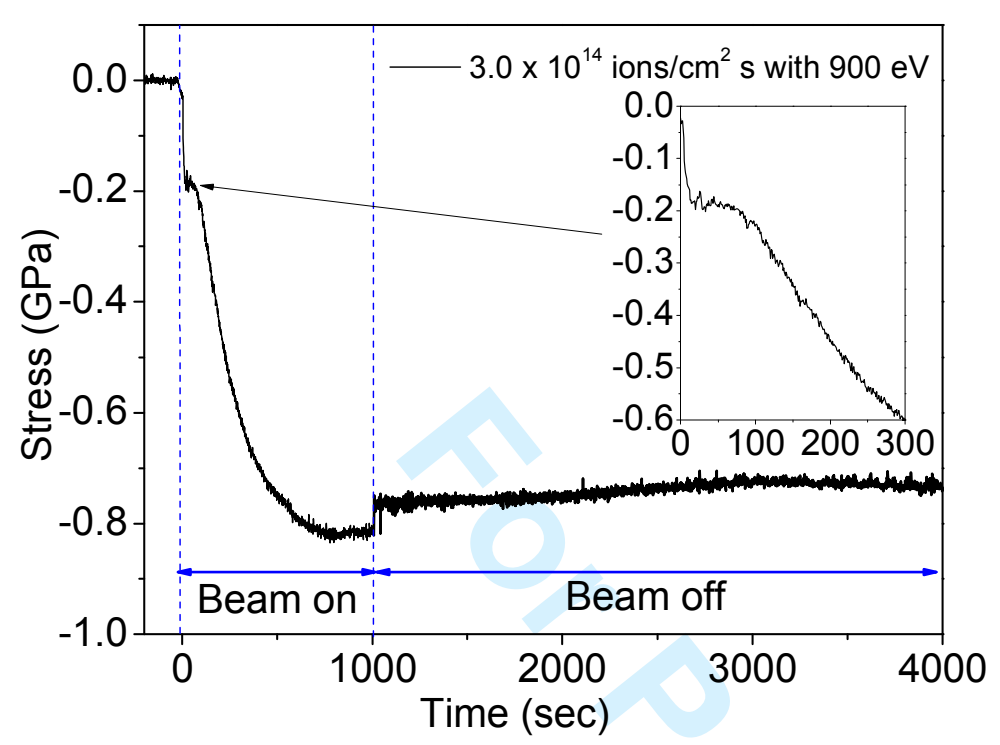

Figure 2

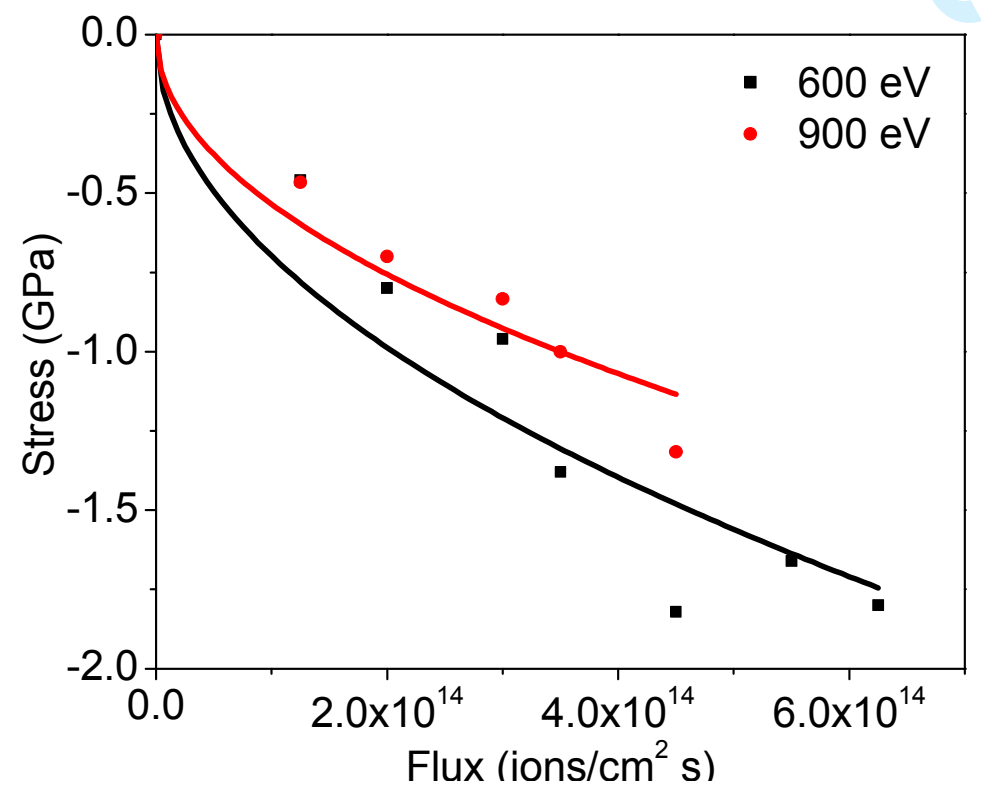


Figure 3
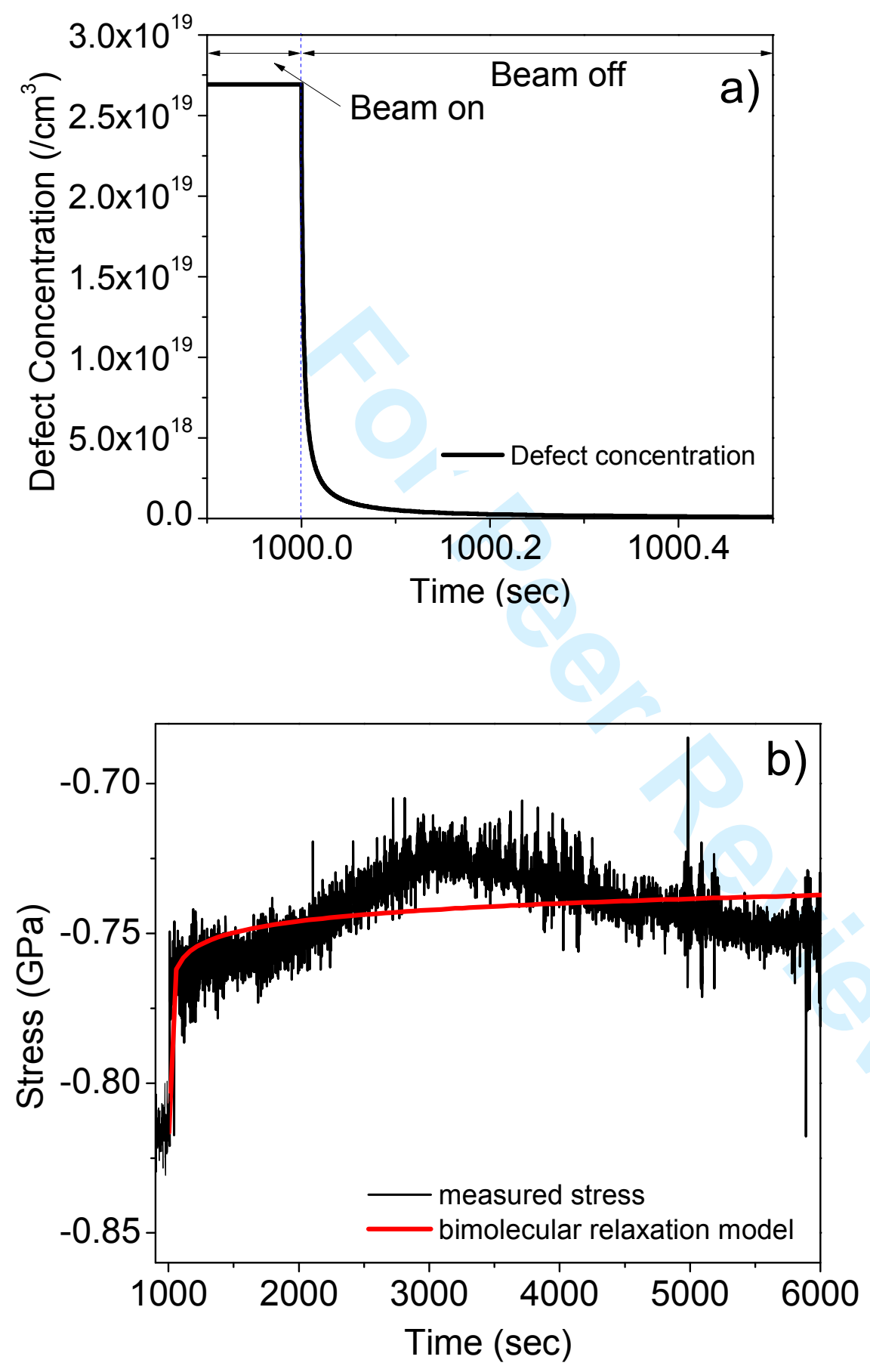
Fig 1. Stress evolution during ion irradiation (in the region labeled beam on, $3.0 \times 1014 \mathrm{ions} / \mathrm{cm} 2 \mathrm{~s}, 900 \mathrm{eV}$ ) and after beam is turned off. Negative stress values are compressive. The inset shows an expanded view of the stress plateau that is attributed to amorphization of the surface. $98 \times 75 \mathrm{~mm}(96 \times 96 \mathrm{DPI})$ 


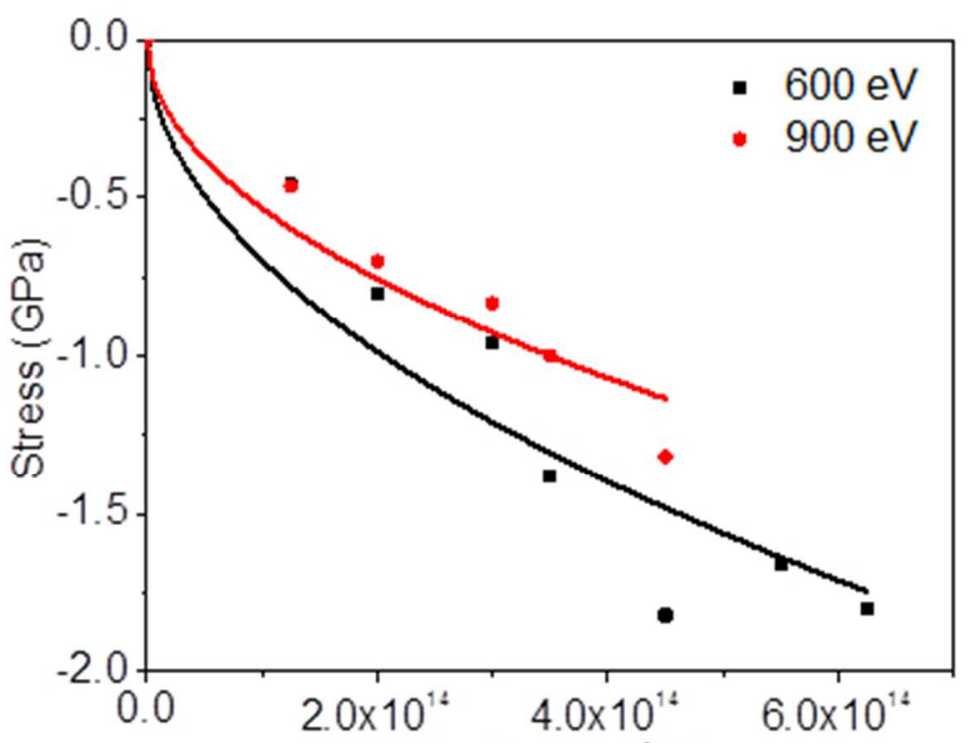

Flux (ions $/ \mathrm{cm}^{2} \mathrm{~s}$ )

Steady-state stress vs flux for ion bombardment with energies of $600 \mathrm{eV}$ and $900 \mathrm{eV}$. Lines are results of model calculations described in text. $102 \times 79 m m$ (96 x 96 DPI) 
1

2

3

4

5

6

7

8

9

10

11

12

13

14

15

16

17

18

19

20

21

22

23

24

25

26

27

28

29

30

31

32

33

34

35

36

37

38

39

40

41

42

43

44

45

46

47

48

49

50

51

52

53

54

55

56

57

58

59

60

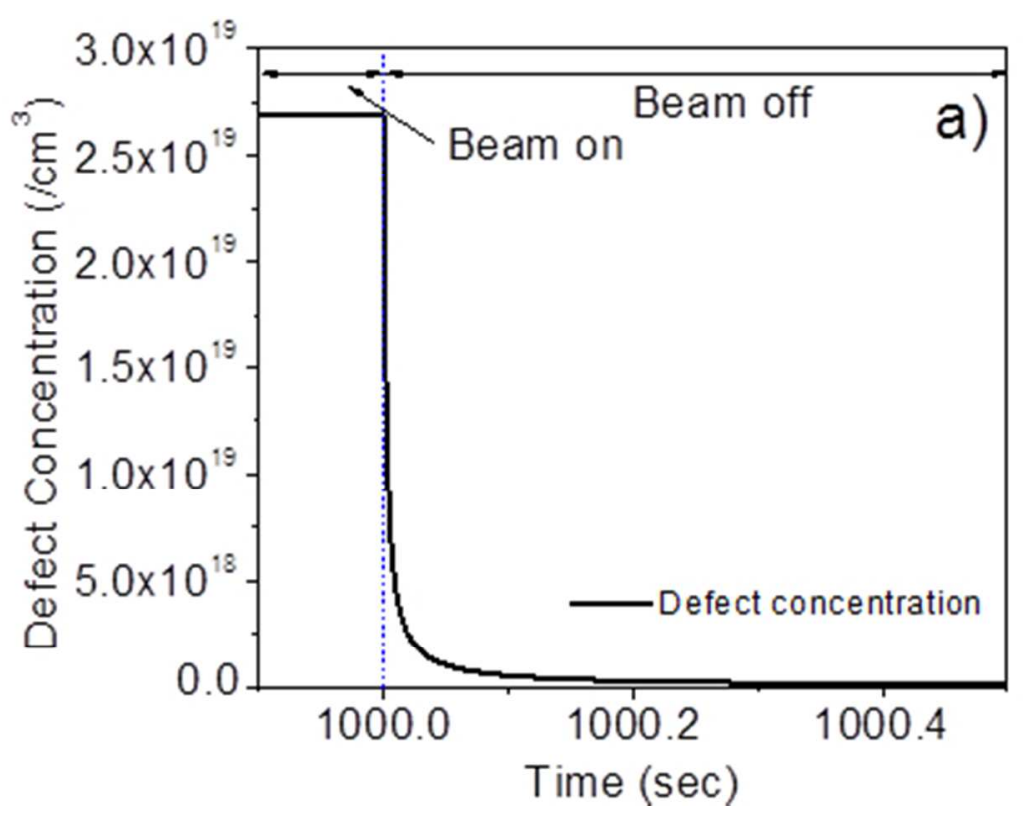

Model calculation of defect concentration in steady-state (beam on) and during relaxation (beam off). $107 \times 80 \mathrm{~mm}(96 \times 96 \mathrm{DPI})$ 


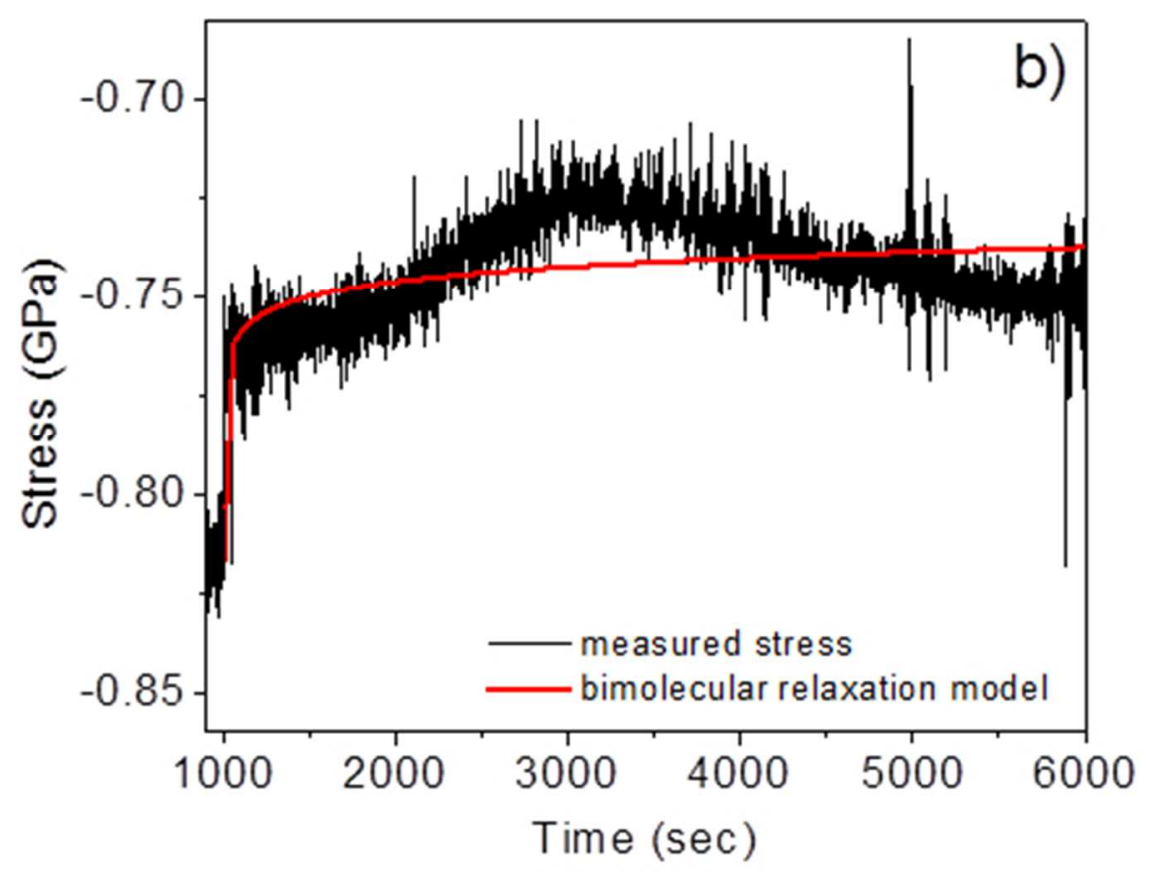

Model calculation of corresponding stress (smooth red line) from defect concentration. The model parameters were chosen to fit the data in figure 1 (shown as the black line for comparison). 